tic fibrosis. J. Clin. Invest. 118:1040-1049.

10. Garred, P., et al. 1999. Association of mannosebinding lectin gene heterogeneity with severity of lung disease and survival in cystic fibrosis. J. Clin. Invest. 104:431-437.

11. Konstan, M.W., et al. 2007. Risk factors for rate of decline in forced expiratory volume in one second in children and adolescents with cystic fibrosis. J. Pediatr. 151:134-139.

12. Frederiksen, B., Koch, C., and Hoiby, N. 1997. Antibiotic treatment of initial colonization with Pseu- domonas aeruginosa postpones chronic infection and prevents deterioration of pulmonary function in cystic fibrosis. Pediatr. Pulmonol. 23:330-335.

13. Rowe, S.M., Accurso, F., and Clancy, J.P. 2007. Detection of cystic fibrosis transmembrane conductance regulator activity in early-phase clinical trials. Proc. Am. Thorac. Soc. 4:387-398.

14. Buranawuti, K., et al. 2006. Variants in mannosebinding lectin and tumor necrosis factor \{alpha\} affect survival in cystic fibrosis. J. Med. Genet. 44:209-214.
15. Harradine, K.A., and Akhurst, R.J. 2006. Mutations of TGFbeta signaling molecules in human disease. Ann. Med. 38:403-414.

16. Mayer-Hamblett, N., Ramsey, B.W., and Kronmal, R.A. 2007. Advancing outcome measures for the new era of drug development in cystic fibrosis. Proc. Am. Thorac. Soc. 4:370-377.

17. Farrell, P.M., et al. 2001. Early diagnosis of cystic fibrosis through neonatal screening prevents severe malnutrition and improves long-term growth. Pediatrics. 107:1-13.

\title{
Hepatic glucose sensing: does flux matter?
}

\author{
Masakazu Shiota1 and Mark A. Magnuson,1,2
}

1Department of Molecular Physiology and Biophysics and 2Vanderbilt Center for Stem Cell Biology, Vanderbilt University School of Medicine, Nashville, Tennessee, USA.

\begin{abstract}
In this issue of the JCI, Denechaud et al. report studies investigating the role of the liver $X$ receptors (LXRs) LXR $\alpha$ and LXR $\beta$ in carbohydrate sensing by the liver (see the related article beginning on page 956). The results of this study, which utilized LXR $\alpha / \beta$ double-KO mice, strongly contradict a recent Nature report that proposed that $\mathrm{LXR} \alpha / \beta$ sense glucose independent of metabolic flux. The reported findings further support a key role for the carbohydrate-responsive element-binding protein (ChREBP) in the regulation of lipogenic genes by glucose and dietary carbohydrates.
\end{abstract}

The liver plays a central and vitally important role in glucose homeostasis by continuously adapting its own metabolism to the availability of nutrients and overall energetic needs of the body. The conversion of carbohydrates and amino acids into fatty acids, a process referred to as lipogenesis, is important for the preservation of excess energy. This metabolic conversion involves the induction of a set of lipogenic genes that have long been known to be regulated by dietary intake, particularly carbohydrates. However, because many lipogenic genes are also regulated by insulin and the concentration of insulin itself is acutely sensitive to changes in blood glucose concentration, only in recent years have we learned how carbohydrate, principally glucose, is able to modulate gene expression in a cellautonomous manner.

The ability of mammalian cells to sense changes in glucose has long been thought

Nonstandard abbreviations used: ACC, acetyl-CoA carboxylase; ChORE, carbohydrate response element; ChREBP, carbohydrate-responsive element-binding protein; FAS, fatty acid synthase; GK, glucokinase; G-6-P, glucose-6-phosphate; L-PK, liver-pyruvate kinase; LXR, liver $\mathrm{X}$ receptor.

Conflict of interest: The authors have declared that no conflict of interest exists.

Citation for this article: J. Clin. Invest. 118:841-844 (2008). doi:10.1172/JCI35137. to depend on changes in metabolic flux, as determined by the specific hexose transporters and hexokinases expressed in a given cell. Hepatocytes express both glucose transporter 2 (GLUT2) and glucokinase (GK; also known as hexokinase 4), both of which exhibit affinities for glucose ( $K_{\mathrm{m}}$ and glucose $\mathrm{S}_{0.5}$, respectively) in the low millimolar range. This enables metabolic flux in hepatocytes to essentially parallel any change in blood glucose concentration (1). In this regard, the liver mimics the pancreatic $\beta$ cell, the prototypical glucose-sensitive cell type. However, functional parallels between these 2 cell types begin to fade beyond this point, due to the very distinct physiological functions of the liver and $\beta$ cell in maintaining glucose homeostasis.

While mammals are thought to sense glucose in a manner that directly reflects glucose flux, other mechanisms that do not rely on catalysis exist in both plants and yeast. For instance, the plant hexokinase HXK1 remains capable of signaling after being mutated in a manner that eliminates all catalytic activity (2). A recent study in Nature by Mitro et al. reported that glucose is able to directly bind to and increase the transcriptional activity of the liver X receptors (LXRs) (3), thereby suggesting that these nuclear receptors also function as glucose sensors in the liver. If true, this would establish a new paradigm for glucose sensing in mammalian cells. The study by Denechaud et al. in this issue of the JCI (4) was directed at exploring this proposed paradigm by assessing the effects of a high-carbohydrate diet on hepatic lipogenesis in mice lacking both LXR $\alpha$ and LXR $\beta$.

\section{SREBP-1c and ChREBP: key players in hepatic lipogenesis}

Over recent years, information has emerged indicating that SREBP-1c and carbohydrate-responsive element-binding protein (ChREBP) both contribute to the regulation of lipogenesis in liver, albeit in very distinct ways. SREBP-1c, the SREBP isoform expressed at high levels in the liver (5), is a member of the basic helix-loop-helix (bHLH) leucine zipper family of transcription factors. This family of transcription factors was originally identified through its role in regulating cholesterol and fatty acid biosynthesis by sterols $(6,7)$. However, SREBP-1c was later found to be regulated by insulin signaling (8). SREBP-1c affects the expression of target genes by binding to sterol response elements, usually located near the promoter region of a target gene. In addition to the regulation of SREBP-1c gene expression by insulin, insulin appears to regulate the cleavage of SREBP-1c, which also directly affects the amount of SREBP-1c in the nucleus (9). SREBP-1 KO mice exhibit profound abnormalities in lipid metabolism (10).

ChREBP, another bHLH transcription factor, also plays a central role in the dietary regulation of hepatic gene expression. This factor was identified by its binding to carbohydrate response elements (ChOREs) in a variety of glucose-respon- 


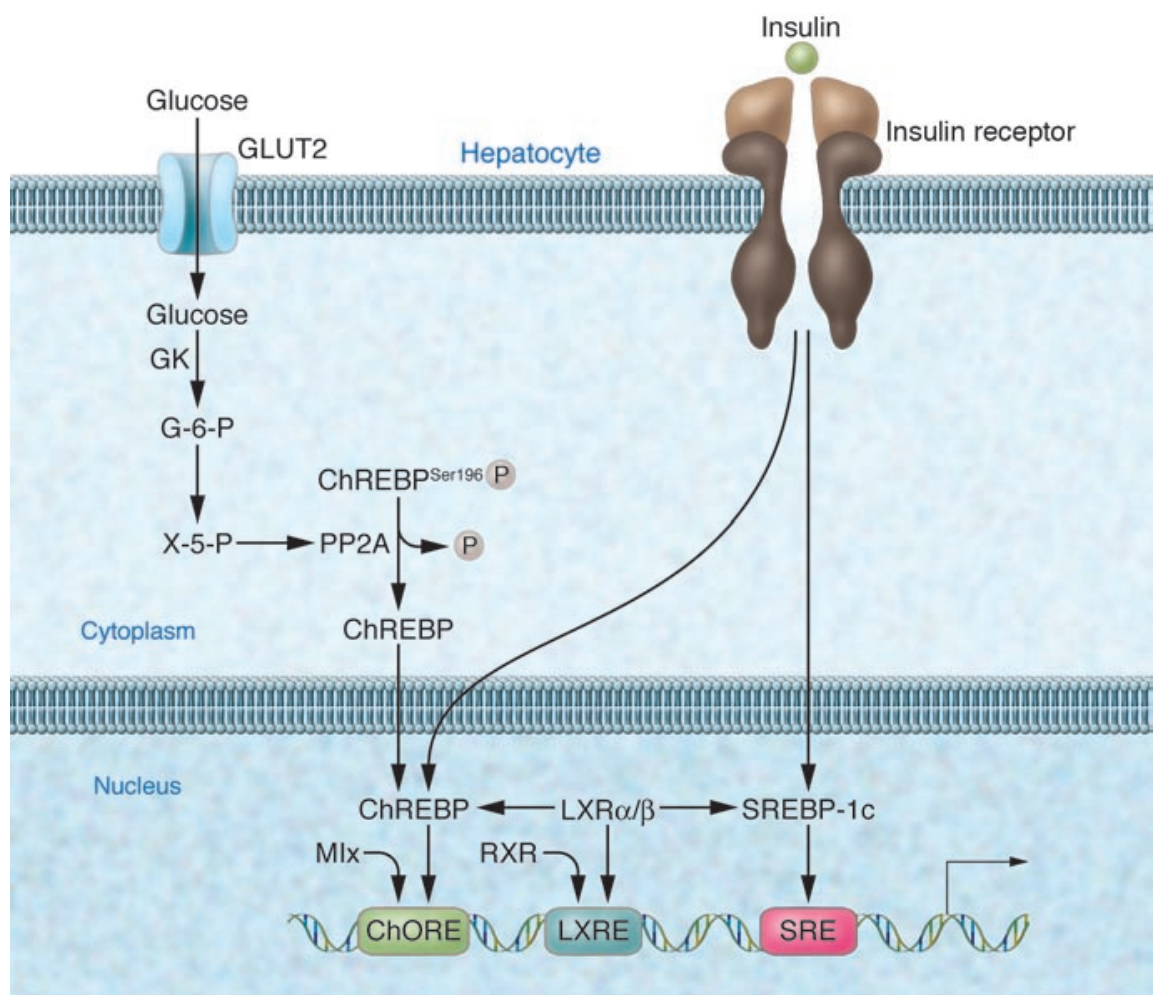

Figure 1

Transcriptional regulation of hepatic lipogenesis by glucose and insulin. In this issue, Denechaud et al. (4) describe a series of studies that strongly challenge a recent report by Mitro et al. (3) suggesting that $L X R \alpha / \beta$ has a direct glucose-sensing function in liver. Denechaud et al. provide strong evidence for ChREBP, not the LXRs, in mediating the effect of glucose on genes in the liver (4). The regulation of hepatic lipogenesis involves several transcriptional regulatory networks. Three transcription factors, ChREBP, LXR $\alpha / \beta$, and SREBP-1c, contribute to this regulation, but in an often incestuous manner. For instance, $L X R \alpha / \beta$ has been found to regulate the transcription of both ChREBP and SREBP-1C. ChREBP, LXR $\alpha / \beta$, and SREBP-1c bind to ChOREs, LXR response elements (LXREs), and sterol response elements (SREs), respectively. Moreover, DNA binding by ChREBP and $L X R \alpha / \beta$ requires heterodimerization with Max-like protein (Mlx) and retinoid $X$ receptor $(\mathrm{RXR})$, respectively. Genes that regulate hepatic lipogenesis have been found to contain 1 or more ChOREs, LXREs, and/or SREs. Some genes, such as for FAS, are regulated by all 3 transcription factors. The authors demonstrate that glucose flux is necessary for regulation of hepatic genes by glucose and that phosphorylation of ChREBP at Ser196 is reciprocally regulated by glucose flux. ChREBP is located in the cytoplasm under low plasma glucose concentration due to the phosphorylation of Ser196. As the plasma glucose level rises, glucose enters hepatocytes and is rapidly equilibrated due to the low- $K_{\mathrm{m}}$ glucose transporter GLUT2. Glucose phosphorylation is then initiated by GK, leading to the formation of xyulose-5-phosphate (X-5-P) by the hexose monophosphate shunt pathway. Increased xyulose-5-phosphate in turn appears to cause dephosphorylation of Ser196 on ChREBP (probably through activation of PP2A), thereby allowing ChREBP to enter the nucleus and affect gene expression. Insulin appears to affect hepatic lipogenesis principally by increasing transcription of both ChREBP and SREBP-1C.

sive, lipogenic genes, including those encoding acetyl-CoA carboxylase (ACC), fatty acid synthase (FAS), and liver-pyruvate kinase $(\mathrm{L}-\mathrm{PK})(11,12)$. Since the initial discovery of ChREBP, this protein has been found to play a central role in the regulation of hepatic genes by glucose. Indeed, mice without this transcription factor exhibit a marked shift in substrate plasm due to its being phosphorylated at Ser196 by protein kinase A. However, at high glucose concentrations, ChREBP becomes dephosphorylated, probably by protein phosphatase $2 \mathrm{~A}(\mathrm{PP} 2 \mathrm{~A})$, thereby stimulating its translocation to the nucleus $(14,15)$ as shown in Figure 1.

\section{Do the LXRs, upstream modulators of ChREBP and SREBP1c, function as glucose sensors?}

LXR $\alpha$ and LXR $\beta$, closely related members of the nuclear hormone receptor superfamily, have also been found to contribute to the regulation of hepatic lipogenesis (16). While first classified as orphan nuclear receptors, both were discovered to bind oxysterols, metabolites of cholesterol, and to regulate genes critical to cholesterol efflux and the conversion of cholesterol to bile acids (17). Moreover, LXR $\alpha$ and LXR $\beta$ have recently been found to bind to regulatory elements in the genes encoding SREBP- $1 \mathrm{c}$ and ChREBP (18), thereby positioning each of these transcription factors to regulate a wide number of genes, some of which encode enzymes involved in fatty acid metabolism (19). All of these responses are abolished in $\mathrm{LXR} \alpha / \beta$ double-KO mice (19).

Recently, Mitro et al. reported that glucose is able to bind and stimulate the transcriptional activity of LXRs (3). This group reported that both D- and L-glucose, as well as glucose-6-phosphate (G-6-P), the initial metabolite of D-glucose, bind to and activate the LXRs, thereby leading to the activation of LXR target genes, including ChREBP, in HepG2 cells (3). On account of these data, Mitro et al. proposed that the LXRs function directly as glucose sensors in the liver. Because the LXRs lie upstream of both SREBP-1c and ChREBP in the glucose and insulin signaling pathways in liver, they are certainly positioned to be able to regulate many downstream genes (Figure 1). However, questions were raised immediately about this study (20), including the fact that the experiments were performed in HepG2 cells, a human hepatocellular carcinoma cell line that responds poorly, if at all, to glucose, and that there is no stereo-specific discrimination between D- and L-glucose. In the model proposed by Mitro et al., glucose would activate the expression of lipogenic genes by the activation of the LXRs. This in turn would increase the expression of both ChREBP and SREBP-1c. However, if this model were true, then the 
ability of glucose to induce the expression of lipogenic genes would be lost in LXR $\alpha / \beta$ double-KO mice.

\section{New evidence against a glucose- sensing function by the LXRs}

The current report by Denechaud et al. (4) provides new insights into the respective roles of ChREBP, SREBP-1c, and the LXRs in the signal transduction pathways involved in regulating lipid synthesis in the liver. The authors performed a series of experiments directed at clarifying the separate roles of ChREBP, SREBP-1c, and $\operatorname{LXR} \alpha / \beta$ in the nutritional regulation of hepatic genes.

First, using C57BL/6J mice that were either fed or fasted, as well as primary hepatocytes from these mice, Denechaud et al. show that LXR activation by the synthetic agonist T0-901317 is not able to overcome the posttranslational modifications of ChREBP mediated by glucose metabolism and that glucose flux is necessary for the transcriptional activation and nuclear localization of ChREBP (4). These studies made use of a phosphospecific antibody developed by the authors that enabled the phosphorylation of ChREBP at Ser196 to be directly monitored. T0-901317, the LXR $\alpha / \beta$-activating agonist, did not affect either the dephosphorylation or nuclear translocation of ChREBP nor the expression of the gene encoding L-PK, which is known to be directly regulated by ChREBP. Finally, the authors demonstrated that refeeding fasted LXR $\alpha / \beta$ double-KO mice with a high-carbohydrate diet increases ChREBP gene expression and the translocation of this protein into the nucleus.

Second, the authors compared the extent to which LXRs contribute to the regulation of 8 hepatic genes that play key roles in glycolysis and lipogenesis by comparing the effects of fasting and refeeding a high-carbohydrate diet in wild-type and LXR $\alpha / \beta$ double-KO mice (4). This experiment revealed that in $\operatorname{LXR} \alpha / \beta$ double$\mathrm{KO}$ mice there was a lack of induction of genes regulated principally by SREBP-1c and LXR $\alpha / \beta$ and that the high-carbohydrate diet-mediated induction of ChREBP expression and its translocation to the nuclei of hepatocytes was unaffected. In interpreting this experiment, the authors dutifully note that there are different regulational patterns. Specifically, L-PK and ACC are principally regulated by ChREBP and stearoyl-CoA desaturase 1
(SCD1) and glyceraldehyde 3-phosphate acyltransferase (GPAT) are under the transcriptional control of SREBP-1c and/or the LXRs, whereas FAS is synergistically regulated by all 3 factors.

Third, the authors examined the regulation by glucose of L-PK and ACC gene expression in hepatocytes from LXR $\alpha / \beta$ double-KO mice (4). These genes, both of which contain well-defined ChOREs, were adequately induced by the addition of glucose, a finding that is directly contrary to what would be expected if LXR $\alpha$ and LXR $\beta$ were acting as hepatic glucose sensors. However, the induction by glucose of both L-PK and ACC did not occur after the cells were treated with an siRNAexpressing recombinant adenovirus that knocked down ChREBP.

Fourth, Denechaud et al. used a fluorescence resonance energy transfer assay to test the ability of glucose and several glucose metabolites to induce the recruitment of several coactivator peptides by the LXR ligand-binding domain (4). This experiment provided another direct test of the model proposed by Mitro et al. (3), namely, that LXR $\alpha$ and $\operatorname{LXR} \beta$ function as hepatic glucose sensors by recruiting transcriptional coactivators in response to increasing concentrations of glucose or its metabolites. While the LXR agonist T0-901317 induced the recruitment of cofactor peptides to both LXR $\alpha$ and LXR $\beta$, as would be expected, neither glucose nor G-6-P, when added directly to this cell-free assay, had any effect. Moreover, by performing cotransfection experiments using chimeric LXR $\alpha / \beta$-Gal4 DNA-binding proteins and a Ga14-responsive luciferase reporter in HepG2 cells (the same cell line used by Mitro et al.), Denechaud et al. showed that glucose had no potentiating effect (4).

When considered together, existing knowledge about the function of ChREBP in the liver and the results of the current study by Denechaud et al. strongly challenge the findings of Mitro et al. and simultaneously greatly strengthen the case for ChREBP being the principal determinant for the regulation of hepatic genes by glucose and dietary carbohydrate. Indeed, these new findings provide very convincing evidence that LXR $\alpha$ and LXR $\beta$ do not have a direct glucose-sensing function in the liver.

So, does metabolic flux really matter for the regulation of hepatic genes by glucose? The answer, as has been thought to be the case for decades, remains yes.

\section{Where to from here?}

While ChREBP, SREBP-1c, and LXRs all play integral roles in the regulation of lipogenesis by the liver, much remains to be learned about how they are regulated, especially by insulin and glucose. In particular, a great deal more remains to be determined about the regulation of ChREBP. Specifically, how does phosphorylation regulate the activity and nuclear transport of this protein; what protein kinases and phosphatases are involved; and do specific metabolites play a role? New and emerging technologies may make such determinations possible. Indeed, only then will our understanding of this intriguing aspect of liver function be complete.

\section{Acknowledgments}

The authors are supported by NIH grant DK60667 (to M. Shiota) and DK42502 and DK72473 (to M.A. Magnuson).

Address correspondence to: Mark A. Magnuson, Center for Stem Cell Biology, Vanderbilt University School of Medicine, 9475 MRB-IV, 2213 Garland Avenue, Nashville, Tennessee 37232, USA. Phone: (615) 3227006; Fax: (615) 322-7236; E-mail: mark. magnuson@vanderbilt.edu.

1. Magnuson, M., and Matschinsky, F. 2004. Glucokinase as a glucose sensor: past, present and future. In Glucokinase and glycemic disease: from basics to novel therapentics. Karger. Basel, Switzerland. $1-17$.

2. Moore, B., et al. 2003. Role of the Arabidopsis glucose sensor HXK1 in nutrient, light, and hormonal signaling. Science. 300:332-336.

3. Mitro, N., et al. 2007. The nuclear receptor LXR is a glucose sensor. Nature. 445:219-223.

4. Denechaud, P.-D., et al. 2008. ChREBP, but not LXRs, is required for the induction of glucoseregulated genes in mouse liver. J. Clin. Invest. 118:956-964.

5. Shimomura, I., Shimano, H., Horton, J.D., Goldstein, J.L., and Brown, M.S. 1997. Differential expression of exons $1 \mathrm{a}$ and $1 \mathrm{c}$ in mRNAs for sterol regulatory element binding protein-1 in human and mouse organs and cultured cells. J. Clin. Invest. 99:838-845.

6. Brown, M.S., and Goldstein, J.L. 1997. The SREBP pathway: regulation of cholesterol metabolism by proteolysis of a membrane-bound transcription factor. Cell. 89:331-340.

7. Brown, M.S., and Goldstein, J.L. 1999. A proteolytic pathway that controls the cholesterol content of membranes, cells, and blood. Proc. Natl. Acad. Sci. U. S. A. 96:11041-11048.

8. Foretz, M., et al. 1999. ADD1/SREBP-1c is required in the activation of hepatic lipogenic gene expression by glucose. Mol. Cell. Biol. 19:3760-3768

9. Hegarty, B.D., et al. 2005. Distinct roles of insulin and liver $\mathrm{X}$ receptor in the induction and cleavage of sterol regulatory element-binding protein-1c. Proc. Natl. Acad. Sci. U. S. A. 102:791-796.

10. Shimano, H., et al. 1999. Sterol regulatory element- 
binding protein-1 as a key transcription factor for nutritional induction of lipogenic enzyme genes. J. Biol. Chem. 274:35832-35839.

11. Bergot, M.O., Diaz-Guerra, M.J., Puzenat, N., Raymondjean, M., and Kahn, A. 1992. Cis-regulation of the L-type pyruvate kinase gene promoter by glucose, insulin and cyclic AMP. Nucleic Acids Res. 20:1871-1877.

12. Shih, H.M., Liu, Z., and Towle, H.C. 1995. Two CACGTG motifs with proper spacing dictate the carbohydrate regulation of hepatic gene transcription. J. Biol. Chem. 270:21991-21997.

13. Burgess, S.C., et al. 2008. Carbohydrate-response element-binding protein deletion alters substrate utilization producing an energy-deficient liver. J. Biol. Chem. 283:1670-1678.

14. Nishimura, M., Fedorov, S., and Uyeda, K. 1994. Glucose-stimulated synthesis of fructose 2,6bisphosphate in rat liver. Dephosphorylation of fructose 6-phosphate, 2-kinase:fructose 2,6bisphosphatase and activation by a sugar phosphate. J. Biol. Chem. 269:26100-26106.

15. Kabashima, T., Kawaguchi, T., Wadzinski, B.E., and Uyeda, K. 2003. Xylulose 5-phosphate mediates glucose-induced lipogenesis by xylulose 5 -phosphate-activated protein phosphatase in rat liver. Proc. Natl. Acad. Sci. U. S. A. 100:5107-5112.

16. Zelcer, N., and Tontonoz, P. 2006. Liver X recep- tors as integrators of metabolic and inflammatory signaling. J. Clin. Invest. 116:607-614.

17. Repa, J.J., and Mangelsdorf, D.J. 2002. The liver X receptor gene team: potential new players in atherosclerosis. Nat. Med. 8:1243-1248.

18. Cha, J.Y., and Repa, J.J. 2007. The liver X receptor (LXR) and hepatic lipogenesis. The carbohydrateresponse element-binding protein is a target gene of LXR. J. Biol. Chem. 282:743-751.

19. Tobin, K.A., et al. 2002. Liver X receptors as insulin-mediating factors in fatty acid and cholesterol biosynthesis. J. Biol. Chem. 277:10691-10697.

20. Lazar, M.A., and Willson, T.M. 2007. Sweet dreams for LXR. Cell Metab. 5:159-161.

\section{Growth hormone resurrects adult human thymus during HIV-1 infection}

Kiki Tesselaar and Frank Miedema

Department of Immunology, University Medical Center Utrecht, Utrecht, The Netherlands.

In conditions of severe $T$ cell depletion, such as HIV-1 infection, limited T cell production by the thymus can thwart the immune response, putting individuals at increased risk of infection with opportunistic pathogens. In this issue of the JCI, Napolitano et al. report, in a prospective, randomized study, that treatment of HIV-1-infected adults with growth hormone may reverse thymic atrophy, as reflected by increased de novo thymic $T$ cell production accompanied by increased peripheral $T$ cell production (see the related article beginning on page 1085). While the long-term immunological and clinical benefits of growth hormone treatment remain unclear, the data suggest a way in which to enhance thymopoiesis and peripheral $T$ cell production in immunodeficient individuals.

$\mathrm{T}$ cell depletion due to (a) chemo- or radiotherapy for the treatment of cancer; (b) myeloablative chemotherapy prior to bone marrow transplantation; (c) immunosuppressive treatment to avoid transplant rejection; or (d) HIV infection can be quite prolonged. These patients may be susceptible to life-threatening viral infections and opportunistic and virus-induced cancers for longer periods of time. Moreover, given the increasing numbers of aged individuals in the human population, age-related loss of immune function is a significant and growing clinical problem. Loss of thymus function and decreasing de novo naive $T$ cell production during aging may be the main cause for failing immunity in the elderly $(1,2)$.

Nonstandard abbreviations used: GH, growth hormone; HAART, highly active antiretroviral therapy; rGH, recombinantGH; TRA@, TCR $\alpha$ locus; TRD@, TCR $\delta$ locus; TREC, TCR rearrangement excision circle.

Conflict of interest: The authors have declared that no conflict of interest exists.

Citation for this article: J. Clin. Invest. 118:844-847 (2008). doi:10.1172/JCI35112.
Peripheral, homeostatic $\mathrm{T}$ cell proliferation may compensate for decreasing and insufficient thymic production of naive $\mathrm{T}$ cells but does not broaden the TCR repertoire. Since for proper $\mathrm{T}$ cell reconstitution, influx of recent thymic emigrants into the $T$ cell pool is required to maintain the naive and memory $T$ cell pool and the TCR repertoire, homeostatic proliferation may result in accelerated aging of the $\mathrm{T}$ cell pool, as reflected by limited TCR diversity, loss of $\mathrm{T}$ cell function, and cellular senescence (3).

Loss of thymic export as a predominant source of $\mathrm{T}$ cell neogenesis with age has been formally proven in the mouse (1). In humans, the association between thymic atrophy and the decline of naive $\mathrm{T}$ cell numbers and TCR rearrangement excision circle (TREC) frequency with age is well documented (1). TRECs are stable DNA circles that are excised from $\mathrm{T}$ cell germline DNA to allow for TCR formation during early $\mathrm{T}$ cell development. Briefly, early in $\mathrm{T}$ cell neogenesis within the thymus, during TCR generation and rearrangement, the TCR $\delta$ locus (TRD@) is excised from within the TCR $\alpha$ locus (TRA@). This excision generates a circular episomal DNA fragment known as a TREC (Figure 1). Thymic emigrants have high concentrations of TRECs, which upon cell division are not replicated but are gradually diluted with every round of division. Increase in TREC density and number has become an accepted indicator of thymic function. Thymic output has been found to be directly related to thymic cellularity (4), but in the mouse there is no evidence for rebound of thymic output as a homeostatic response to severe depletion of the peripheral $\mathrm{T}$ cell pool. In humans, evidence for thymic rebound has been claimed based on increased thymic mass or exceedingly high TREC frequencies observed during immune reconstitution. However, this issue remains controversial (5).

\section{Causes of thymic atrophy}

Gradual, age-associated thymic atrophy is believed to be caused by declining levels of several hormones, including human growth hormone $(\mathrm{GH})$ and its mediator IGF-1, as well as the cytokine IL-7 (1). In the mouse, a relationship between IL-7 and thymic T cell output, as well as between IL-7 and peripheral T cell division, are well established (6). Studies in humans and nonhuman primates, however, have mainly shown effects of IL-7 on peripheral $\mathrm{T}$ cell division (7-9).

$\mathrm{GH}$ is produced by a large variety of cell types, including thymocytes, thymic 